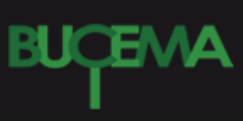

Bulletin du centre d'études médiévales d'Auxerre | BUCEMA

Hors-série $n^{\circ} 10 \mid 2016$

L'origine des sites monastiques : confrontation entre la terminologie des sources textuelles et les données archéologiques

Monasterium, cella, abbatia... Enquête sur les différents termes désignant les communautés religieuses au haut Moyen Âge $\left(\mathrm{V}^{\mathrm{e}}\right.$-milieu $\mathrm{IX}^{\mathrm{e}}$ siècle) et leur signification

\title{
Michèle Gaillard
}

\section{OpenEdition \\ Journals}

\section{Édition électronique}

URL : https://journals.openedition.org/cem/14474

DOI : $10.4000 /$ cem. 14474

ISSN : 1954-3093

Éditeur

Centre d'études médiévales Saint-Germain d'Auxerre

\section{Référence électronique}

Michèle Gaillard, «Monasterium, cella, abbatia... Enquête sur les différents termes désignant les communautés religieuses au haut Moyen Âge ( $v$ e-milieu Ixe siècle) et leur signification », Bulletin du centre d'études médiévales d'Auxerre / BUCEMA [En ligne], Hors-série $n^{\circ} 10$ | 2016, mis en ligne le 09 décembre 2016, consulté le 04 mars 2023. URL : http://journals.openedition.org/cem/14474 ; DOI : https://doi.org/10.4000/cem.14474

Ce document a été généré automatiquement le 4 mars 2023.

\section{c) (7)(2)}

Creative Commons - Attribution - Pas d'Utilisation Commerciale - Partage dans les Mêmes Conditions 4.0 International - CC BY-NC-SA 4.0

https://creativecommons.org/licenses/by-nc-sa/4.0/ 


\section{Monasterium, cella, abbatia... Enquête sur les différents termes désignant les communautés religieuses au haut Moyen Âge ( $\mathrm{v}^{\mathrm{e}}$-milieu $\mathrm{IX}^{\mathrm{e}}$ siècle) et leur signification}

\section{Michèle Gaillard}

Abréviations :

AA SS : Acta Sanctorum, par les Bollandistes, nouvelle éd. 1863-1868.

BHL : Bibliotheca hagiographica Latina antiquae et mediae aetatis ediderunt Socii Bollandiani, 2 vol., Bruxelles, 1898-1901, Supplementum, 1911, Novum Supplementum, 1986.

HF : GRÉGOIRE DE TOURS, Libri Historiarum X, in MGH, Scriptores Rerum Merowingicarum, I, 1, éd. B. KRUSCH, Hanovre, 1941.

Lib. in Glor : GRÉGOIRE DE TOURS, Liber in Gloria Martyrum, in MGH, Scriptores Rerum Merowingicarum, I, 2, éd. B. KRUSCH, Hanovre, 1885, p. 34-112.

Lib. Vit. Patrum : GRÉGOIRE DE TOURS, Liber Vitae Patrum, in MGH, Scriptores Rerum Merowingicarum, I, 2, éd. B. KRUSCH, Hanovre, 1885, p. 211-294.

Liber Virt. sancti Iuliani : GRÉGOIRE DE TOURS, Liber Virtutum sancti Iuliani, in MGH, Scriptores Rerum Merowingicarum, I, 2, éd. B. KRUsch, Hanovre, 1885, p. 112-134. MGH : Monumenta Germaniae Historica inde ab anno christi quingentesimo usque ad annum millesimumet quingentesimum edidit societas aperiendis fontibus rerum germanicarum medii aevi.

MIGNE, PL : Abbé Jacques-Paul MIGNE, Patrologiae Cursus Completus, sive bibliotheca universalis..., Paris, 1850. 
TCCG : Topographie chrétienne des cités de la Gaule, 16 tomes (en 17 volumes), Paris, 1986-2014.

1 Toute personne fréquentant quelque peu les sources écrites du haut Moyen Âge a été un jour confrontée à différents termes utilisés pour désigner les communautés religieuses. Les termes monasterium/monasteriolum, coenobium, cella/cellula, voisinent à la même époque, voire chez les mêmes auteurs, mais on trouve aussi les termes casa, claustrum, conventum dans quelques textes ainsi que le terme abbatia, qui a donné naissance au français abbaye, aujourd'hui quasi-synonyme de monastère. Rappelons que le terme capitulum n'était pas alors utilisé pour désigner les communautés de chanoines, mais seulement l'assemblée des moines ou des chanoines à la première heure (prime), où on lisait un chapitre (capitulum) de la règle ${ }^{1}$.

Cette enquête porte donc sur un certain nombre de mots (abbatia, casa, cella, cellula, claustrum, coenobium, congregatio, conventum, monasterium, xenodochium ${ }^{2}$ ), qui sont susceptibles de désigner les communautés religieuses qu'elles soient monastiques ou canoniales ${ }^{3}$, tout en gardant à l'esprit deux questions sous-jacentes :

- ces différents termes désignent-ils des réalités différentes, dans leur architecture, leur organisation, leur importance (subjective ou objective) ou bien sont-ils quasiment synonymes et seulement le reflet des spécificités de chaque auteur ou de chaque période?

- y a-t-il une évolution de la signification de ces termes entre le $v^{e}$ et le $\mathrm{IX}^{\mathrm{e}}$ siècle, soit vers une restriction du sens ou bien vers un usage plus large de termes ayant au départ une signification précise?

Bien sûr cette enquête n'est pas exhaustive, mais elle s'appuie sur un nombre suffisant de sources pour être significative ; une première approche a été faite grâce à l'index des fascicules de la Topographie chrétienne des cités de la Gaule. Cet index permet de retrouver les mots employés dans un nombre important et très varié de sources pour désigner les communautés religieuses installées dans l'espace urbain. L'approche est donc incomplète, puisqu'il n'est pas question des implantations rurales, mais surtout parce qu'elle dépend des citations choisies par les auteurs des notices pour chaque établissement. Il est néanmoins probable que le hasard de ces choix nous laisse percevoir un usage proche de la réalité, d'autant que les plus anciennes mentions avant le milieu du viII siècle - ont été systématiquement listées.

Ces données ont été ensuite complétées par l'étude du vocabulaire employé dans les règles monastiques : Institutions de Cassien, Règles de Césaire d'Arles, Règle du Maître, Règle de saint Benoît, Règles des saints Pères et également par la Vie des Pères du Jura qui comporte bien des aspects normatifs ${ }^{4}$ - et, pour la période carolingienne, par la Vie de Benoît d'Aniane ${ }^{5}$ et les Annales de Saint-Bertin ${ }^{6}$. J'ai aussi soumis certains des volumes des Monumenta Germaniae Historica, les Scriptores rerum merowingicarum ${ }^{7}$, les Diplomata $^{8}$ et les Concilia à une interrogation sur les termes abbatia et monasterium pour la période $\mathrm{du} \mathrm{VI}^{\mathrm{e}}$ au milieu du $\mathrm{IX}^{\mathrm{e}}$ siècle. 


\section{Monasterium}

5 C'est le mot le plus fréquent, qui vient de loin devant tous les autres avec cinquantehuit occurrences dans les notices de la TCCG; le tableau 1 permet de ventiler sa fréquence dans les sources étudiées.

Tab. 1 - Les occurrences du mot monasterium (et variantes)

\begin{tabular}{|c|c|c|}
\hline Sources & $\begin{array}{l}\text { Nombre } \\
\text { d'occurrences }\end{array}$ & Remarques \\
\hline $\begin{array}{l}\text { Institutions de Cassien } \\
\text { (fin } \mathrm{IV}^{\mathrm{e}} \text {-début } \mathrm{V}^{\mathrm{e}} \mathrm{s} \text {.) }\end{array}$ & 89 & Texte très long. \\
\hline $\begin{array}{l}\text { Règles des Pères } \\
\left(\mathrm{V}^{\mathrm{e}} \mathrm{s} .\right)\end{array}$ & 24 & Ensemble de 6 règles. \\
\hline $\begin{array}{l}\text { Règle du Maître } \\
\text { (fin } V^{e} / \text { déb. } \mathrm{VI}^{\mathrm{e}} \mathrm{s} . \text { ) }\end{array}$ & 253 & La plus longue de toutes les règles. \\
\hline $\begin{array}{l}\text { Règles de Césaire } \\
\left(\mathrm{VI}^{\mathrm{e}} \mathrm{s} .\right)\end{array}$ & 51 & Deux règles et lettres. \\
\hline $\begin{array}{l}\text { Règle de saint Benoît } \\
\text { (Vers 530) }\end{array}$ & 74 & \\
\hline $\begin{array}{l}\text { Vie des Pères du Jura } \\
\text { (Début } \mathrm{VI}^{\mathrm{e}} \mathrm{s} . \text { ) }\end{array}$ & 41 & \\
\hline $\begin{array}{l}\text { Vie de Benoît d'Aniane } \\
\text { (821) }\end{array}$ & 80 & \\
\hline $\begin{array}{l}\text { Annales de Saint-Bertin } \\
\mathrm{IX}^{\mathrm{e}} \mathrm{s} \text {. }\end{array}$ & 46 & Dont 2 monasteriolum dans un sens péjoratif. \\
\hline $\begin{array}{ll}\text { MGH } & \\
\text { Scriptores } & \text { rerum } \\
\text { merowingicarum } & \end{array}$ & 1094 & Dont 165 dans les œuvres de Grégoire de Tours ( $† 594)$. \\
\hline $\begin{array}{lr}\text { MGH } & \\
\text { Diplomata } & \text { regum } \\
\text { francorum e } & \text { stirpe } \\
\text { merovingica } & \\
\text { (Éd. Kölzer, 2001) }\end{array}$ & 430 & $\begin{array}{l}\text { Nouvelle édition comprenant uniquement les } \\
\text { diplômes royaux mais intégrant les mentions d'un } \\
\text { grand nombre de mentions de diplômes perdus. }\end{array}$ \\
\hline $\begin{array}{l}\text { MGH } \\
\text { Diplomata }\end{array}$ & 1049 & $\begin{array}{l}\text { Diplômes de Pépin le Bref, Carloman, Charlemagne, } \\
\text { Lothaire I }{ }^{\mathrm{er}} \text { et Lothaire II ; dont } 35 \text { monasteriolum sans } \\
\text { sens péjoratif. }\end{array}$ \\
\hline $\begin{array}{l}\text { MGH } \\
\text { Concilia }\end{array}$ & $\begin{array}{l}\text { mérovingiens : } \\
37 \\
\text { carolingiens : } 398\end{array}$ & Jusqu'en 859. \\
\hline
\end{tabular}


Malgré son caractère incomplet, le sondage effectué dans les MGH grâce au moteur de recherche en ligne confirme que le terme monasterium vient naturellement sous la plume des auteurs mérovingiens et carolingiens pour désigner des communautés religieuses. Ce qui justifie amplement l'emploi du terme « monastère » en français. Mais force est de constater que l'emploi de ce terme n'implique pas que l'on ait affaire à des moines, en tout cas au $\mathrm{IX}^{\mathrm{e}}$ siècle : la plus grande partie des moines de Saint-Denis, monasterium sancti Dyonisii, en 832, revendiquent d'être, depuis toujours, des chanoines, de même que ceux de Tours: les diplômes délivrés par Louis le Pieux en faveur de Saint-Martin de Tours en 816 et 832 montrent que les frères de ce qu'on continue d'appeler un monasterium, ont réussi à faire admettre, sans grande difficulté, semble-til, leur statut de chanoines. Dans le diplôme d'immunité de 816, il est question des clercs qui servent Dieu ${ }^{10}$ et en 832 des frères du monastère ${ }^{11}$. Dans un diplôme de 869, Charles le Chauve qualifie Saint-Arnoul de Metz de monasterium canonicorum ${ }^{12}$, monastère de chanoines, tant le terme s'impose pour désigner la personnalité juridique d'une communauté religieuse vivant dans des bâtiments spécifiques et ayant des revenus fonciers.

\section{Coenobium}

Bien qu'étant bien moins fréquent et moins régulièrement employé que le mot monasterium, le mot coenobium désigne aussi un monastère. On le voit dans les citations de la TCCG (tableau 2) comme synonyme de cella et de monasterium (en grisé).

Tab. 2 - Coenobium (et variantes) d'après les notices de la TCCG

\begin{tabular}{|l|l|l|l|}
\hline Vocabulaire & Réf TCCG & Citation & Contexte et source \\
\hline & $\begin{array}{l}\text { Arles, III, 15, } \\
\text { p. 84; XVI, 15, } \\
\text { p. } 45\end{array}$ & $\begin{array}{l}\text { in suburbano } \\
\text { coenobio }\end{array}$ & $\begin{array}{l}\text { = in suburbana insula... monasterium } \\
\text { (Vie de Césaire, I, 12, p. 161). }\end{array}$ \\
\hline $\begin{array}{l}\text { Auxerre, VII,, } \\
21-25, \\
\text { p. 62-63 }\end{array}$ & $\begin{array}{l}\text { cellam } \\
\text { coenobium }\end{array}$ & $\begin{array}{l}\text { (charte de l'évêques Palladius, 637). } \\
\text { avec trois églises (Julien, Ferréol, Martin); }\end{array}$ \\
\hline $\begin{array}{l}\text { Beauvais, XIV, } \\
2, \text { p. } 140\end{array}$ & $\begin{array}{l}\text { coenobium, } \\
\text { oratorium }\end{array}$ & $\begin{array}{l}\text { Vie d'Ansbert } \\
=\text { Oratorium monasterium } \\
\text { dans le testament d'Anségise } \\
=\text { olim puellarum coenobium } \\
\text { (diplôme perdu de Charles le Chauve). }\end{array}$ \\
\hline $\begin{array}{l}\text { Coenobium / } \\
\text { coenubium / } \\
\text { cenobium }\end{array}$ & $\begin{array}{l}\text { Bourges, VI, } \\
13, \text { p. 24 }\end{array}$ & $\begin{array}{l}\text { basilica, ecclesia, } \\
\text { monasterium, } \\
\text { coenobium }\end{array}$ & $\begin{array}{l}\text { Miracula Austrigisilii (rédigés entre 741 et 751). } \\
\text { Tombeau de saint Ausregisile. }\end{array}$ \\
\hline
\end{tabular}




\begin{tabular}{|c|c|c|}
\hline $\begin{array}{l}\text { Cahors, VI, 11, } \\
\text { p. } 63\end{array}$ & $\begin{array}{l}\text { coenobium sancti } \\
\text { viri }\end{array}$ & $\begin{array}{l}\text { = suum monasterium; fondé par l'év Didier (vita } \\
\text { Desiderii). }\end{array}$ \\
\hline $\begin{array}{l}\text { Clermont, VI, } \\
21, \text { p. } 38 ; 23, \\
\text { p. } 38\end{array}$ & $\begin{array}{l}\text { puellarum } \\
\text { cenobium }\end{array}$ & $\begin{array}{l}\text { Absence de coenobium de femmes avant la } \\
\text { construction par Praejectus d'un monasterium } \\
\text { consacré aux puellis (Passio Praejecti, fin VII }{ }^{\mathrm{e}} \text {. }\end{array}$ \\
\hline $\begin{array}{l}\text { Laon, XIV, 1, } \\
\text { p. } 169\end{array}$ & $\begin{array}{l}\text { infra } \quad \text { claustra } \\
\text { coenobii }\end{array}$ & $=$ monasterium (Vita Sadalbergae). \\
\hline $\begin{array}{l}\text { Soissons, XIV, } \\
1, \text { p. } 55\end{array}$ & intra coenubii septa & $\begin{array}{l}\text { Privilège de l'évêque Drauscius pour Sainte- } \\
\text { Marie de Soissons (666-667). }\end{array}$ \\
\hline $\begin{array}{l}\text { Orléans, VIII, } \\
6, \text { p. } 94\end{array}$ & $\begin{array}{l}\text { coenobium } \\
\text { sanctarum } \\
\text { monacharum }\end{array}$ & Vie de saint Eucher. \\
\hline
\end{tabular}

8 L'usage fait par les auteurs de règles est très disparate : 51 occurrences chez Cassien (contre 89 pour monasterium), aucune dans les Règles des Pères, deux seulement dans la Règle du Maître, aucune chez Césaire, une seule chez Benoît, 16 dans la Vie des Pères du Jura. Il n'y a là aussi aucun indice qui permette de penser qu'il y ait une nuance de sens entre le monasterium et le coenobium même si l'étymologie est différente : le monasterium venant du moine, qui est seul (monos en grec) et le coenobium désignant la vie communautaire. L'influence orientale a pu jouer chez Cassien dans le choix fréquent de ce terme ; l'absence du terme dans les règles de Pères pourrait conduire à minimiser l'influence orientale sur ces règles...

\section{Casa}

9 Le troisième tableau donne des occurrences de casa, qui, pour la plupart, ne désignent pas des monastères, mais des habitations de solitaires.

Tab. 3 - Casa d'après les notices de la TCCG

\begin{tabular}{|l|l|l|l|}
\hline Vocabulaire & Réf TCCG & Citation & Contexte et source \\
\hline & $\begin{array}{l}\text { Clermont, } \\
\text { VI, 12, } \\
\text { p. } 36\end{array}$ & $\begin{array}{l}\text { casa cui culmo culmina pressa } \\
\text { forent : cabane dont le toit } \\
\text { est couvert de chaume }\end{array}$ & $\begin{array}{l}\text { Maison de l'épitaphe rédigée par S. Apollinaire (Ep. } \\
\text { VII, 17 p. 75). Selon Grégoire de Tours a } \\
\text { érigé un monastère tout près. }\end{array}$ \\
\hline Casa & $\begin{array}{l}\text { Trèves, } \\
\text { I, 5, p. 26 }\end{array}$ & $\begin{array}{l}\text { casa servorum Dei: cabane } \\
\text { des serviteurs de Dieu }\end{array}$ & $\begin{array}{l}\text { Augustin (Conf. XII, 15) rapporte le récit } \\
\text { d'un fonctionnaire impérial (386). }\end{array}$ \\
\hline $\begin{array}{l}\text { XII, } \\
\text { p. 21-22 }\end{array}$ & $\begin{array}{l}\text { casa Dei sancti Martini, quem } \\
\text { domnus } \\
\text { archiepiscopus custos presse } \\
\text { videtur }\end{array}$ & $\begin{array}{l}\text { Diplôme de Pépin Ir du 23 mai 753: un } \\
\text { Monastère dirigé par Boniface selon } \\
\text { Mostert? }\end{array}$ \\
\hline
\end{tabular}


À cela deux exceptions :

- la première mention est celle d'un groupement d'ermites dans un édifice suffisamment humble pour qu'on y applique le terme « casa »;

- pour la seconde, il doit s'agir d'une communauté desservant l'église Saint-Martin d'Utrecht. Est-ce un monastère comme le suggère $\mathrm{M}$. Mostert ${ }^{13}$ ou la communauté des clercs desservant l'église d'Utrecht, rien ne permet de trancher.

Dans les Scriptores rerum merowingicarum, seulement deux textes emploient ce mot dans un contexte religieux :

- la Vie de sainte Bathilde parle des casas peculiares (cellules?) construites par Bathilde pour son monastère ${ }^{14}$, mais le mot ne désigne pas le monastère en son entier ;

- les miracles de saint Austregisèle, évêque de Cahors, font état de la destruction de casas Dei, mais rien n'indique qu'il s'agisse spécifiquement de monastères ${ }^{15}$.

Ce mot n'est pas employé dans les textes questionnés (règles en particulier) sauf dans le Règle du Maître, où il est question des casae monasterii (62 et 86) mais au sens de curtis, domaine, ferme...

\section{Cella et cellula}

Les mots cella et cellula qui apparaissent là (tableau 4) synonymes (cf. pour Radegonde) correspondent souvent à des habitations individuelles - ou presque pour Hilaire et son frère - près d'une église ou à l'intérieur même du monastère.

Tab. 4 - Cella et cellula comme cellules d'après les notices de la TCCG

\begin{tabular}{|c|c|c|c|}
\hline \multirow[t]{2}{*}{ Vocabulaire } & Réf TCCG & Citation & Contexte et source \\
\hline & $\begin{array}{l}\text { Arles, III, } 4, \\
\text { p. } 81\end{array}$ & $\begin{array}{l}\text { eiusdem cellae erat cellae } \\
\text { eius coniuncta }\end{array}$ & $\begin{array}{l}\text { Cellule d'Hilaire jouxtant les cellae } \\
\text { des autres clercs. }\end{array}$ \\
\hline & $\begin{array}{l}\text { Arras, XIV, } \\
2, \text { p. } 95\end{array}$ & $\begin{array}{l}\text { cellula tegumenta; cum lecto } \\
\text { cellola }\end{array}$ & $\begin{array}{l}\text { Lieu où est mort Vaast et qui fut } \\
\text { ensuite épargné par l'incendie qui } \\
\text { consuma la domus (Vie de saint } \\
\text { Vaast, attribuée à Jonas de Bobbio, } \\
\text { 9-10). }\end{array}$ \\
\hline & $\begin{array}{l}\text { Bourges, } \\
\text { VI, } 4, \text { p. } 21\end{array}$ & $\begin{array}{l}\text { cellulam iuxta ecclesiam in } \\
\text { superiori moenia civitatis }\end{array}$ & $\begin{array}{l}\text { Cellule de l'ascète Amandus (Vita S. } \\
\text { Amandi (SRM V, p. 433). }\end{array}$ \\
\hline & $\begin{array}{l}\text { Bourges, } \\
\text { VI, p. } 25\end{array}$ & cellula Ebregiseli & Cellule d'un reclus (Vita Eligii, p. 731). \\
\hline & $\begin{array}{l}\text { Cahors, } \\
\text { XVI, } \\
\text { p. } 71\end{array}$ & $\begin{array}{l}\text { reclusit se in cellula quae } \\
\text { erat iuxta ecclesiam }\end{array}$ & $\begin{array}{l}\text { Cellule de l'évêque Ambroise, qui } \\
\text { communique avec l'ecclesia (Vita } \\
\text { Ambrosii). }\end{array}$ \\
\hline & $\begin{array}{l}\text { Clermont, } \\
\text { VI, 13, p. } 36\end{array}$ & cellula sancti Martii & $\begin{array}{l}\text { Habitation de l'ermite Martius, } \\
\text { creusée dans le rocher; y organise } \\
\text { un monastère. }\end{array}$ \\
\hline
\end{tabular}




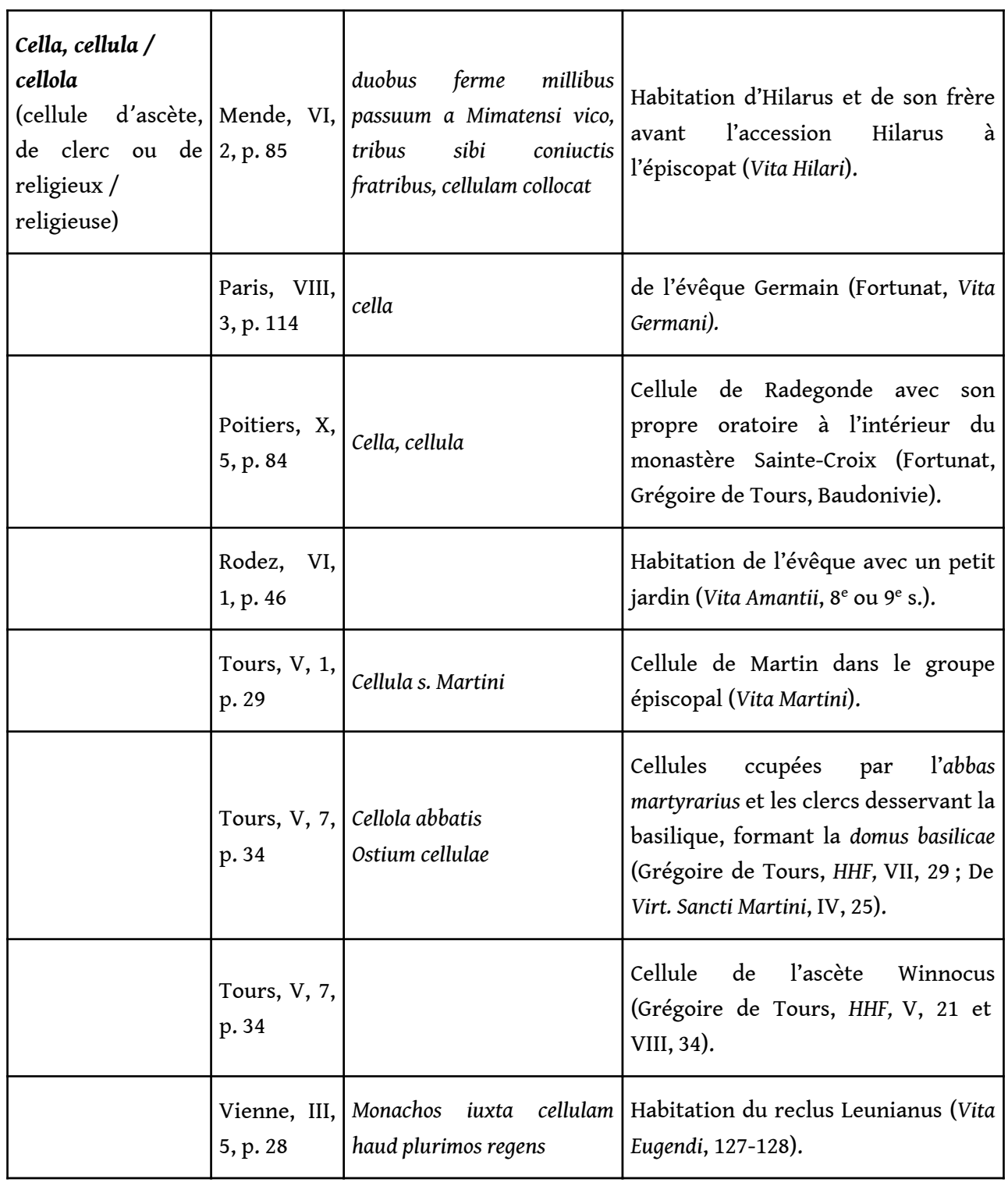

Mais cella et cellula peuvent avoir un tout un autre sens : de toute évidence celui de monastère (tableau 5).

Tab. 5 - Cella et cellula comme habitations collectives à travers les notices de la TCCG

\begin{tabular}{|l|l|l|l|}
\hline Vocabulaire & Réf TCCG & Citation & Contexte et source \\
\hline & $\begin{array}{l}\text { Angoulême, } \\
\text { X, 2, p. 50 }\end{array}$ & $\begin{array}{l}\text { Cellulam sibi aedificavit in qua, collectis } \\
\text { pauchis monachis, in oratione } \\
\text { morabatur assidue }\end{array}$ & $\begin{array}{l}\text { Grégoire de Tours, à } \\
\text { propos d'Eparchius, abbé } \\
\text { de Saint-Cybard (HF, VI, } \\
8) .\end{array}$ \\
\hline Amiens, XIV, & Cellula puellarum religiosarum & $\begin{array}{l}\text { Grégoire de Tours la situe } \\
\text { à l'endroit où saint } \\
\text { Martin avait partagé son } \\
\text { manteau (Vita. Martini, } \\
\text { p. 148). }\end{array}$ \\
\hline
\end{tabular}




\begin{tabular}{|c|c|c|c|}
\hline & $\begin{array}{l}\text { Auxerre, VIII, } \\
21-25, \\
\text { p. } 62-63\end{array}$ & Cellam sive coenobium & $\begin{array}{l}\text { avec trois églises dédiées } \\
\text { à Julien, Ferréol, Martin } \\
\text { (charte de l'év Palladius, } \\
637 \text { ). }\end{array}$ \\
\hline \multirow[t]{5}{*}{$\begin{array}{l}\text { Cella, cellula/ } \\
\text { cellola } \\
\text { (habitation } \\
\text { collective pour } \\
\text { moines, } \\
\text { religieuses ou } \\
\text { clercs) }\end{array}$} & $\begin{array}{l}\text { Bourges, VI, } \\
11, \text { p. } 23\end{array}$ & $\begin{array}{l}\text { abba ordinaretur in basilicam sancti } \\
\text { Simphoriani quam memoratus pontifex } \\
\text { fabricaverat ante conspectum muri } \\
\text { Biturigi. Nec tamen monachos quos } \\
\text { prius congregaverat relinquens, sed } \\
\text { instituens eis praepositum, ipse } \\
\text { utrasque cellulas gubernabat. }\end{array}$ & $\begin{array}{l}\text { À propos de l'acète } \\
\text { Agustus, abbé de Saint- } \\
\text { Martin puis de Saint- } \\
\text { Symphorien (Grégoire de } \\
\text { Tours, Glor. Conf., p. } 347 \text { ). }\end{array}$ \\
\hline & $\begin{array}{l}\text { Le Mans, V, 9, } \\
\text { p. } 52\end{array}$ & Cella sancte marie & $\begin{array}{l}\text { Privilège de l'évêque } \\
\text { Aglibertus (683); diplôme } \\
\text { de Louis le Pieux (836). }\end{array}$ \\
\hline & $\begin{array}{l}\text { Marseille, III, } \\
11, \text { p. 131-132 }\end{array}$ & Ad cellulam in finibus Massiliensis urbis & $\begin{array}{l}\text { Vie d'Hilaire évêque du } \\
\text { Gévaudan (BHL 3910): il } \\
\text { visite la cellula et oublie } \\
\text { son manteau dans le } \\
\text { cubiculum monachorum. }\end{array}$ \\
\hline & $\begin{array}{l}\text { Tours, V, 11, } \\
\text { p. } 36\end{array}$ & $\begin{array}{l}\text { Parva cellula ibique paucas collegens } \\
\text { monachas }\end{array}$ & $\begin{array}{l}\text { Fondée par Monegundis } \\
\text { selon Grégoire de Tours } \\
(V P, X I X, 2) \text {. }\end{array}$ \\
\hline & $\begin{array}{l}\text { Troyes, VIII, } \\
\text { 7, p. } 79\end{array}$ & Cella domni Bobini & $\begin{array}{l}\text { Diplôme de Charles le } \\
\text { Chauve (Tessier, p. 291) } \\
\text { pour désigner Montier-la- } \\
\text { Celle; nom de l'abbé } \\
\text { devenu év de Troyes au } \\
\text { milieu du } 8^{\mathrm{e}} \mathrm{s} \text {. }\end{array}$ \\
\hline $\begin{array}{l}\text { autre sens de } \\
\text { cellula }\end{array}$ & $\begin{array}{l}\text { Le Mans, V, 4, } \\
\text { p. } 51\end{array}$ & Cellula sancti Martini & $\begin{array}{l}=\text { oratorium, situé près de } \\
\text { la cathédrale (Actus du } \\
\text { Mans). }\end{array}$ \\
\hline
\end{tabular}

Il ne semble pas qu'il y ait une différence entre les deux : la cellula n'est pas plus petite que la cella. Grégoire de Tours doit préciser parva cellula, pour suggérer que l'établissement fondé par Monegundis était petit et ne pouvait contenir que quelques religieuses: paucas monachas. Grégoire n'emploie jamais le mot cella au sens de monastère, il ne l'emploie que deux fois dans tout son œuvre: une pour désigner la pièce garde-manger, cella penaria, où sont entreposées dans un premier temps les reliques de saint Saturnin de Toulouse ${ }^{16}$ et l'autre pour désigner la pièce proche de l'église saint Julien (de Brioude), où un malade installe son lit ${ }^{17}$. Quant aux 104 occurrences de cellula dans l'œuvre de Grégoire, elles font apparaître aussi bien le sens d'édifice que celui plus précis de monastère : le sens de monastère dans son œuvre ne peut être déduit que des précisions qui l'accompagnent. Il emploie également ce mot pour des oratoires, des tombeaux et aussi des cellules de moines. 
Chez Grégoire, comme dans les autres sources mentionnées dans la TCCG, ni cellula ni cella ne suffisent à désigner un monastère ou une communauté de clercs, seul le contexte ou la connaissance qu'on a par ailleurs du lieu peuvent permettre de trancher. Il faut donc être prudent: si un texte de l'époque mérovingienne parle d'une cellula à propos d'un lieu, sans autre précision, même si ce lieu est attesté plus tard comme occupé par des moines au $\mathrm{IX}^{\mathrm{e}}$ siècle par exemple, il ne faut pas en conclure que ce fut dès l'origine un monastère.

Dans les règles et vies normatives questionnées, les occurrences de cella/cellula sont évidemment nombreuses et les deux mots semblent interchangeables (tableau 6).

Tab. 6 - Cella et cellula dans les règles et vies normatives

\begin{tabular}{|c|c|c|c|c|}
\hline \multirow[b]{2}{*}{ Sources } & \multicolumn{3}{|c|}{ Nombre d'occurrences } & \multirow[b]{2}{*}{ Remarques } \\
\hline & Cella & Cellula & 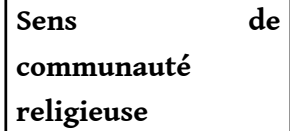 & \\
\hline $\begin{array}{l}\text { Institutions de } \\
\text { Cassien }\end{array}$ & 24 & 23 & 1 & $\begin{array}{l}\text { cellasque monasteria conspeximus... } \\
\text { Ailleurs cella/cellula synonymes: cellule du } \\
\text { moine ou pièce du monastère. }\end{array}$ \\
\hline $\begin{array}{l}\text { Règles des } \\
\text { Pères }\end{array}$ & 7 & 12 & 7 & $\begin{array}{l}\text { Ex : } \\
\text { - cellam ut paradisium habeat... } \\
\text { - tota dilectio vestra in cellula demoretur... } \\
\text { Dans la règle de Macaire }(7 / 8 \text { occurrences) } \\
\text { et la } 3^{\text {e }} \text { règle des Pères }(4 / 6) \text { qui dérive de la } \\
\text { précédente. }\end{array}$ \\
\hline $\begin{array}{l}\text { Règle } \quad d u \\
\text { Maître }\end{array}$ & 7 & 5 & 1 & $\begin{array}{l}\text { per diversorum cellas et monasteria } \\
\text { hospitantes... } \\
\text { À propos des moines gyrovagues. }\end{array}$ \\
\hline $\begin{array}{l}\text { Règles de } \\
\text { Césaire }\end{array}$ & 4 & 2 & $1 ?$ & $\begin{array}{l}\text { cellula monasterii... } \\
\text { cella + génitif au sens de pièce du monastère } \\
\text { (cellier en } V 30,7 \text {; parloir en } V 65,2 \text { ). }\end{array}$ \\
\hline $\begin{array}{l}\text { Règle de saint } \\
\text { Benoît }\end{array}$ & 6 & 0 & 1 & $\begin{array}{l}\text { per diversorum cellas... } \\
\text { À propos des moines gyrovagues. }\end{array}$ \\
\hline $\begin{array}{l}\text { Vie des Pères } \\
\text { du Jura }\end{array}$ & 3 & 8 & 2 (cellula) & \\
\hline $\begin{array}{l}\text { Vie de Benoît } \\
\text { d'Aniane }\end{array}$ & 6 & 0 & 6 & $\begin{array}{l}\text { dont cellam monasterii pour désigner le } \\
\text { monastère dans son ensemble. }\end{array}$ \\
\hline Total & 57 & 60 & 16 à 19 & \\
\hline
\end{tabular}

Cependant il faut souligner deux exceptions notables : l'absence d'emploi du mot cellula dans la Règle de saint Benoît et dans la Vie de Benoît d'Aniane; ces deux textes ont été 
rédigés à quatre siècles d'intervalle et dans des contrées différentes (Italie et Gaule) ; on pourrait considérer que le vocabulaire de la première a pu influencer le rédacteur de la seconde si l'usage du mot cella n'était pas fort différent dans les deux textes. Dans la Règle de saint Benoît, à une exception près - empruntée à la Règle du Maitre de toute évidence ${ }^{18}$-, le mot cella désigne des pièces ou édifices du monastère alors que dans la Vie de Benoît d'Aniane le mot désigne toujours une communauté religieuse, que ce soit celle, modeste, des débuts d'Aniane ou celle de Gellone ${ }^{19}$.

Soulignons cependant que les emplois de cella/cellula pour désigner les monastères sont rares, comparés aux quatre-vingts occurrences du mot monasterium dans les mêmes textes. L'emploi du mot cellula pour désigner le monastère est encore plus rare que celui du mot cella et semble ressortir essentiellement d'un auteur, celui de la Règle de Macaire, reprise dans la Règle des Pères. Césaire l'emploie, mais uniquement dans l'expression cellula monasterii, ce que les traducteurs rendent par bâtiment du monastère ; le mot a donc un sens concret proche de l'emploi qu'il fait de cella ailleurs. Dans la Vie des Pères du Jura, le mot cella n'est jamais employé pour désigner les monastères, mais trois fois - dont deux associées à l'adjectif peculiaris - pour désigner les cellules ${ }^{20}$; seulement deux occurrences de cellula, au sens de monastère, peuvent y être relevées ${ }^{21}$.

Les termes cella et cellula pour désigner les monastères sont donc attestés à toutes les époques, mais d'un emploi relativement rare. Il est impossible de dire si l'usage de ces termes implique une différence de statut ou une différence de perception du lieu de la part de l'auteur ; autrement dit les mots cella/cellula sont-ils strictement synonymes de monastère ? Les Institutions de Cassien et la Règle du Maître emploient des expressions qui associent les deux mots: s'agit-il d'une façon de désigner deux types de communautés ou bien d'une redondance dont sont friands les auteurs médiévaux? Cassien emploi l'expression cellasque monasteria pour montrer qu'il a examiné les usages liturgiques (nombre de psaumes chantés) dans différents lieux et qu'il a constaté une grande diversitée ${ }^{2}$; le Maître l'emploie pour désigner les lieux où passent les moines gyrovagues : per diversarum cellas et monasteria hospitantes ${ }^{23}$. Il s'agit donc de montrer la diversité des lieux, mais probablement pas d'introduire une différence de taille, de forme, d'organisation, d'autant que Benoît ne retient de l'expression du maître que : per diversorum monasteria ${ }^{24}$.

L'examen de ces règles et de ces deux textes hagiographiques fortement normatifs ne permet guère, une fois encore, que d'insister sur la nécessité d'apporter la plus grande attention au contexte d'utilisation de ces termes fortement polysémiques. Il est difficile de discerner une évolution chronologique puisque le terme cella au sens de monastère est utilisé au $\mathrm{v}^{\mathrm{e}}$ siècle (Règle de saint Benoît) et au $\mathrm{IX}^{\mathrm{e}}$ siècle (Vie de Benoît d'Aniane); cependant il s'est imposé suffisamment longtemps pour qu'on retrouve dans le nom de bien des monastères ensuite. Il faudrait examiner un échantillonnage de textes hagiographiques et diplomatiques sur plusieurs siècles pour déterminer pourquoi et comment cet usage s'impose dans la toponymie monastique, parfois même aux côtés de celui de monastère, comme à Montier-la-Celle près de Troyes.

\section{Claustrum}

Continuons notre enquête par un mot latin qui a eu une grande fortune en français pour désigner un monastère, ou une partie du monastère, le mot claustrum. On le 
trouve peu dans les notices de la TCCG, car il ne sert jamais à désigner l'édifice clos, le monastère en son entier (tableau 7); il en désigne évidemment le mur de clôture ou l'espace enclos, mais pas spécifiquement en contexte monastique et toujours au pluriel.

Tab. 7 - Claustrum dans les notices de la TCCG

\begin{tabular}{|l|l|l|l|}
\hline Vocabulaire & Réf TCCG & Citation & Contexte et source \\
\hline & $\begin{array}{l}\text { Laon, } \\
\text { XIV, } \\
1, \text { p. } 169\end{array}$ & infra claustra ... coenobii & $\begin{array}{l}\text { L'abbesse Salaberge déambule dans le } \\
\text { jardin à l'intérieur de la clôture du } \\
\text { monastère (Vita Sadalbergae). }\end{array}$ \\
\hline Claustrum & $\begin{array}{l}\text { Limoges, } \\
\text { VI, } \\
2, \text { p. } 76\end{array}$ & claustra & $\begin{array}{l}\text { Clôture de l'ensemble basilical de Saint- } \\
\text { Martial. }\end{array}$ \\
\hline $\begin{array}{l}\text { Vienne, } \\
\text { XVI, } \\
5, \text { p. } 318\end{array}$ & $\begin{array}{l}\text { disruptae sunt catenae et reserata } \\
\text { sunt claustra (les chaînes se } \\
\text { brisèrent et les barrières } \\
\text { s'ouvrirent) }\end{array}$ & $\begin{array}{l}\text { appelée monasterium dans un acte } \\
\text { probablement forgé (par Adon ?) après } \\
831 .\end{array}$ \\
\hline
\end{tabular}

Dans les règles, le mot est peu employé : pas du tout par Cassien, qui ne fait allusion à aucune clôture ou mur, pas plus d'ailleurs que Césaire, dont le contenu de la Règle des Vierges n'autorise pourtant aucun doute sur la vie cloîtrée des religieuses. Il est employé une fois seulement par le Maître et deux fois par Benoît en association: claustra monasterii. Les Règles des Pères ne l'utilisent pas et la Vie des Pères du Jura ne l'utilise pas pour désigner la clôture du monastère et seulement quatre fois : une fois dans un sens métaphorique, deux fois au sens de clôture d'une cellule particulière et une fois pour désigner l'enfermement d'une possédée $e^{25}$. Nulle part le mot septa ne vient le remplacer dans ces règles bien qu'il soit utilisé dans d'autres textes (cf. tab. 2 pour Soissons). La Vie de Benoît d'Aniane ne l'utilise qu'une fois, mais dans un autre sens encore, qui ne semble donc émerger qu'à l'époque carolingienne, celui de cloître architectural $^{26}$.

\section{Congregatio, conventus et xenodochium}

Le mot congregatio est assez régulièrement employé dans les textes étudiés : 22 occurrences chez Cassien, 63 dans la Règle du Maître, 9 fois chez Césaire, 25 fois chez Benoît, 5 fois dans les Règles des Pères, 4 fois dans la Vie des Pères du Jura et 2 fois dans la Vie de Benoît d'Aniane. Il peut être employé seul, mais, la plupart du temps, il est assorti d'une précision: congregatio monachorum, congregatio fratrum. Assez curieusement, il n'apparaît qu'une seule fois dans les notices de la TCCG à propos d'une congregatio mulierum à Mayence : domum Domni et sanctae Mariae virginis ibi congregavi sanctam congregationem mulierum peut-on lire dans une charte interpolée au $\mathrm{XII}^{\mathrm{e}}$ siècle. Même si on ne peut douter de l'usage de ce mot bien avant, il est évident qu'il désigne l'ensemble des religieux rassemblés et non le bâtiment - même si cela implique son existence -, aussi les auteurs des notices ont-ils préféré citer des sources employant des mots au sens plus concrets comme monasterium, cella, coenobium. 
à conventus, qui, dans les textes narratifs non spécifiquement religieux, a le sens d'assemblée - ainsi vingt-six occurrences dans les Annales de Saint-Bertin -, il prend à peu près le sens de congregatio quand il est employé dans les règles. Employé trois fois seulement dans la Règle du Maître, il semble toutefois avoir un sens moins fort et moins institutionnel que congregatio et désigner seulement l'ensemble des frères ou une assemblée des frères, proche de son sens laïc. Benoît ne l'emploie qu'une fois, pour désigner l'assemblée de la communauté, lors du chapitre, semble-t-il ${ }^{27}$. Il n'y a pas d'autres mentions dans les règles étudiées, ni dans la Vie de Benoît d'Aniane. En revanche, la Vie des Pères du Jura emploie deux fois le mot conventiculum pour désigner les offices nocturnes (nocturnis conventiculis) et du matin (matutinis conventiculis), donc là aussi avec le sens d'assemblée et non au sens institutionnel ${ }^{28}$.

Quelques mots pour finir, ou presque, sur le terme xenodochium, qui, a lui seul, ne peut permettre d'identifier un monastère, mais qui y est souvent associé comme le montre le tableau 8 (grisé).

Tab. 8 - Xenodochium d'après les notices de la TCCG

\begin{tabular}{|c|c|c|c|}
\hline Vocabulaire & Réf TCCG & Citation & Contexte et source \\
\hline & $\begin{array}{l}\text { Angers, V, } \\
6, \text { p. } 77\end{array}$ & Xenodochia & En relation avec le monastère Saint-Julien. \\
\hline & $\begin{array}{l}\text { Autun, IV, } \\
7, \text { p. } 43\end{array}$ & $\begin{array}{l}\text { Monasterium atque } \\
\text { xenodochium } \\
\text { Francorum }\end{array}$ & Lettres de Grégoire le Grand. \\
\hline & $\begin{array}{l}\text { Auxerre, } \\
\text { VIII, } \\
6, \text { p. } 56\end{array}$ & $\begin{array}{l}\text { Xenodochium matris } \\
\text { ecclesie }\end{array}$ & Institution dépendant de la cathédrale. \\
\hline & $\begin{array}{l}\text { Auxerre, } \\
\text { VIII, } \\
14, \text { p. } 60\end{array}$ & $\begin{array}{l}\text { Xenodochium } \\
\text { pauperum }\end{array}$ & $\begin{array}{l}\text { Don de l'évêque Desiderius pour le } \\
\text { xenodochium dépendant de la basilique } \\
\text { Saint-Germain. }\end{array}$ \\
\hline & $\begin{array}{l}\text { Chalon-sur- } \\
\text { Saône, IV, } \\
4, \text { p. } 72\end{array}$ & $\begin{array}{l}\text { Exsenodochium } \\
\text { leprosorum et } \\
\text { basilica }\end{array}$ & $\begin{array}{l}\text { Grégoire de Tours (Glor. Conf., 85); } \\
\text { probablement Saint-Marcel. }\end{array}$ \\
\hline \multirow[t]{4}{*}{$\begin{array}{l}\text { Xenodochiolum / } \\
\text { senodociolum }\end{array}$} & $\begin{array}{l}\text { Clermont, } \\
\text { VI, } \\
22, \text { p. } 38\end{array}$ & $\begin{array}{l}\text { Xenodochium qui in } \\
\text { loco Columbarius } \\
\text { dicitur }\end{array}$ & $\begin{array}{l}\text { Passio Preajecti ; = Monasterium Colombariense } \\
\text { (libellus)? }\end{array}$ \\
\hline & $\begin{array}{l}\text { Le Mans, V, } \\
15, \text { p. } 54\end{array}$ & $\begin{array}{lr}\text { Sinodochium } & \text { in } \\
\text { honorem } & \text { sancti } \\
\text { Martini } & \end{array}$ & $\begin{array}{l}=\text { basilica = monasterium (Testament de } \\
\text { l'évêque Bertrichramnus). }\end{array}$ \\
\hline & $\begin{array}{l}\text { Lyon, IV, } \\
10, \text { p. } 31\end{array}$ & $\begin{array}{l}\text { Xenodochium in } \\
\text { Lugdunensi urbe }\end{array}$ & $\begin{array}{l}\text { Fondé par le roi Childebert, canon du } \\
\text { concile d'Orléans. }\end{array}$ \\
\hline & $\begin{array}{l}\text { Paris, VIII, } \\
\text { 5, p. } 114\end{array}$ & Exinodochium & $\begin{array}{l}\text { Projet d'Eloi mais remplacé par monastère } \\
\text { de femmes selon la Vita Eligii. }\end{array}$ \\
\hline
\end{tabular}




\begin{tabular}{|l|l|l|l|}
\hline & $\begin{array}{l}\text { Poitiers, X, } \\
4, \text { p. 82 }\end{array}$ & $\begin{array}{l}\text { Sinodoxium } \\
\text { pauperum }\end{array}$ & Fondé par l'évêque Ansoald (Testament). \\
\hline $\begin{array}{l}\text { Reims, XIV, } \\
\text { p. 44-45 }\end{array}$ & Xenodochia & Construits par Attolus (épitaphe, vers 530). \\
\hline
\end{tabular}

Hospices pour les malades, les étrangers et les pauvres, les xenodochia semblent avoir été des institutions urbaines, gérées par ceux qui devaient faire preuve de charité, les moines mais aussi les évêques et leur clergé. La présence d'un xenodochium n'implique pas la présence d'une communauté monastique, mais quand on n'a pas d'autre renseignement et que l'établissement n'est pas en relation avec la cathédrale, l'hypothèse d'une communauté de moines ou de moniales le desservant est légitime. $\mathrm{Ce}$ terme n'est pas employé dans les règles étudiées. La Vie des Pères du Jura l'emploie au sens d'hôtellerie - pour les étrangers donc - et aussi plus étrangement au sens de dortoir ${ }^{29}$.

\section{Abbatia}

Terminons par ce terme alors inusité, mais qui présente un grand intérêt, puisqu'il a donné en français le terme abbaye, quasiment synonyme de monastère. Il n'y a aucune occurrence de ce terme dans les règles examinées. L'index de la TCCG ne donne qu'une occurrence extraite de la chronique de Frédégaire à propos de l'abbé Leodebodus de Saint-Aignan d'Orléans: il est élevé à l'honneur sublime de l'abbatiat (abbatiae sublimatum honore $)^{30}$. Il désigne donc la charge d'abbé et non le bâtiment, ni même la communauté religieuse. Dans le sondage effectué dans les $M G H$, le mot abbatia apparaît fréquemment, mais presque toujours pour désigner la charge d'abbé et non la personnalité juridique, ni l'édifice ; dans ce dernier cas, on emploie le mot monasterium ou encore cella, coenobium.

Le mot n'est employé qu'une fois par Grégoire de Tours pour désigner l'office abbatial ${ }^{31}$. Le terme n'est pas utilisé par Frédégaire; on le trouve donc au total quinze fois, toujours dans le même sens, à une exception près, la Vie de saint Faron, évêque de Meaux, rédigée à la fin $\mathrm{du} \mathrm{IX}^{\mathrm{e}}$ siècle, où il est employé trois fois pour désigner une abbaye comme nous l'entendons aujourd'hui ${ }^{32}$.

Dans les diplômes mérovingiens, on note deux emplois au sens de charge abbatiale ${ }^{33}$ et dans les diplômes carolingiens étudiés on compte seulement seize occurrences du terme, la plupart du temps dans des diplômes faux, donc rédigés tardivement ou bien au sens de charge abbatiale ${ }^{34}$. Quelques mentions montrent une évolution du sens $d u$ mot vers celui de dotation foncière du monastère, comme dans un diplôme de Charlemagne de 775, où l'immunité conférée à l'Église de Metz s'étend aux monasteria vel castella vicos pagos parrochias vel abbatias, que ad ipsum pontificem aspicere videntur ${ }^{35}$ ou bien dans un de Lothaire II de 858, où il est question d'un bénéfice obtenu par son médecin ex abbatia quae Mariculas vocatur ${ }^{36}$. La seule mention d'une abbatia dans un sens concret non équivoque se trouve dans un diplôme de Lothaire de 849 , où il est question d'une abbatia cum suis cellulis pertinentes ${ }^{37}$; encore faudrait-il établir quel sens donner au mot cellula... 
31 C'est aussi à l'époque carolingienne qu'apparaissent d'autres occurrences d'abbatia au sens actuel d'abbaye, en particulier dans les actes d'un concile tenu en avril 859 in abbatia sanctorum Geminorum ${ }^{38}$ et dans les Annales de Saint-Bertin - rédigées par l'évêque de Troyes, Prudence, de 835 à 861, puis, jusqu'en 882, par Hincmar de Reims -, qui comportent vingt-sept occurrences du mot abbatia. Mais, la plupart du temps, le contexte suggère qu'il s'agit des charges abbatiales ainsi que du revenu et des terres qui leur sont attachées, comme à l'année 837 dans l'énumération des terres donnée par Louis le Pieux à son fils Charles : omnes videlicet episcopatus, abbatias, comitatus, fiscos et omnia intra predictos fines consistentia ${ }^{39}$. Dans quatre cas, seulement, le mot est employé dans un sens qui peut être considéré comme synonyme d'abbaye, en particulier pour la première fois à l'année 867, donc de la plume d'Hincmar: Quo patrato negotio, Karolus synodum apud Trecas 8. Kalendas Novembris auctoritate Nicolai papae indicit, et causa venandi ac expendendi a autumnale tempus in abbatia Sancti Vedasti et in Audriaca villa ac circum circa morandi disponit ${ }^{40}$. En revanche, dans le récit concernant cette même année, on trouve le mot monasterium pour désigner Saint-Denis : Paschas Domini in monasterio sancti Dyonisii celebravit ${ }^{41}$. Les deux mots semblent donc devenir parfois synonymes.

L'évolution du sens du mot abbatia semble donc commencer à l'époque carolingienne, d'abord par un glissement vers le sens de revenus et biens attachés à la charge abbatiale, puis, dans la seconde moitié du Ixe siècle, vers le bâtiment et la communauté dont on a la charge et auxquels sont attachés des revenus, l'abbaye.

Il conviendrait d'examiner les sources du milieu du $\mathrm{Ix}^{\mathrm{e}}$ jusqu'au $\mathrm{xl}^{\mathrm{e}}$ siècle pour saisir le rythme ultérieur de cette évolution. Mais c'est une autre histoire...

\section{Conclusion}

$\mathrm{Au}$ terme de cette enquête on peut donc distinguer, tout d'abord, toute une série de termes dont les significations ne semblent pas changer $d u v^{e}$ au IXe siècle :

- des termes aux sens voisins et dont la signification et le caractère concret, à la fois architectural et institutionnel, ne posent pas de problème : monasterium et coenobium employés indifféremment par les mêmes auteurs, mais pas par tous ;

- des termes polysémiques, cella et cellula, servant à la fois à désigner le contenant et le contenu, le monastère et les pièces s'y trouvant, mais qui peuvent aussi être utilisés dans d'autres contextes pour désigner un oratoire ou/et un tombeau ;

- un terme, casa, employé rarement pour désigner un monastère, avec probablement une notion d'humilité.

On a repéré également des termes désignant non les bâtiments, mais les hommes (ou femmes) qui y vivent religieusement : congregatio avec un sens institutionnel fort, me semble-t-il, et conventus, au sens institutionnel moins fort, mais promis à un bel avenir au Moyen Âge classique et au-delà.

Il existe également des termes pouvant être associés à un monastère, mais ne permettant pas d'en affirmer l'existence: claustrum et xenodochium, qui ne sont quasiment pas employés dans les règles étudiées.

37 Enfin, une place particulière doit être accordée au mot abbatia, qui n'est jamais employé dans les règles étudiées, mais est fréquent dans bien d'autres textes pour désigner non pas le bâtiment, ni la communauté, mais la charge abbatiale assortie, à l'époque carolingienne, de la cession d'une partie des revenus de l'établissement : ceux 
qui reçoivent alors l'abbatia sont alors le plus souvent des laïcs ou des séculiers et ne vivent que rarement dans l'abbaye. Pour ce terme, on perçoit au $\mathrm{IX}^{\mathrm{e}}$ siècle une évolution vers le sens plus concret actuel du mot d'abbaye. Il convient donc d'examiner avec attention les contextes historiques et textuels de ces termes, en particulier des mots monasterium et abbatia avant de les traduire ou de les utiliser pour caractériser une communauté religieuse ou le bâtiment qui l'abrite.

\section{NOTES}

1. Capitulare monasticum, $817, \S 69$, MGH, Capitularia, 1, éd. A. BORETIUS, Hanovre, 1883, p. 348 : Ut ad capitulum primitus martyrologium legatur et dicatur versus quo silentium solvatur, deinde regula aut homelia quaelibet legatur, novissime « tu autem Domine » dicatur.

2. Ces mots ont été sélectionnés grâce à l'index des volumes de la Topographie chrétienne des cités de la Gaule; cf. F. PRÉVOT, M. GAILLARD et N. GAUTHIER (éd.), Topographie chrétienne des cités de la Gaule des origines au milieu du VIII siècle, t. 16/1 (Quarante ans d'enquête, 1972-2012. Images nouvelles des villes de la Gaule), t. 16/2 (Quarante ans d'enquête, 1972-2012. Christianisation et espace urbain: atlas, tableaux, index), Paris, 2014.

3. Rappelons que jusqu'au $\mathrm{IX}^{\mathrm{e}}$ siècle, la différence entre les deux styles de vie n'est pas encore véritablement établie, ce qui amena Alcuin (†796) à reconnaître l'existence de communautés du troisième degré : tertius gradus qui hos duos (= inter canonicos et monachos) variatur, intermédiaires entre les chanoines des cathédrales, théoriquement soumis à l'évêque, et les monastères dirigés par des abbés, cf. MGH, Epistolae karolini aevi, II, éd. E. DÜMMLER, Berlin, 1895, p. 416, nº 258.

4. JEAN CASSIEN, Institutions cénobitiques, éd. trad. J.-C. GUY, Paris, 2001 ( $2^{\mathrm{e}}$ éd.) ; CÉSAIRE D’ARLES, CEuvres monastiques, éd. et trad. A. DE VoGÜÉ et J. COURREAU, Paris, 1988 ; La Règle du Maitre, éd. et trad. A. DE VoGÜÉ, 3 vol., Paris, 1964 ; La Règle de saint Benoît, éd. et trad. A. DE VOGÜÉ et J. NEUfVILLE, 6 vol., Paris, 1971 ; Les Règles des saints Pères, éd. et trad. A. DE voGüÉ, 2 vol., Paris, 1982 ; Vie des Pères du Jura, éd. et trad. F. MARTINE, Paris, 2004.

5. ARDON, Vie de Benoit d'Aniane, introduction et notes par P. BONNERUE, trad. F. BAUMES (La vie de saint Benoît d'Aniane, par saint Ardon, son disciple, traduite sur le texte même du cartulaire d'Aniane, Paris, 1910) revue et corrigée par A. DE VOGÜÉ, Bégrolles-en-Mauges, 2001.

6. Annales de Saint-Bertin, éd. F. GRAT, J. VIELLIARD et S. CLÉMENCET, Paris, 1964.

7. http://www.dmgh.de/de/fs1/object/display.html?sortIndex=010:020; dans l'analyse, il faudra se souvenir du fait qu'il ne s'agit pas seulement de textes écrits à l'époque mérovingienne et du fait que ces volumes contiennent aussi nombre de récits rédigés à l'époque carolingienne.

8. http://www.dmgh.de/de/fs1/object/display.html?sortIndex $=030$; jusqu'à Lothaire II inclus ; mais cette série ne comporte pas les diplômes de Charles le Chauve (Recueil des actes de Charles II le Chauve, roi de France, éd. G. TESSIER, 3 vol., Paris, 1943), ni ceux de Louis le Pieux, pour lequel on ne dispose pas encore d'édition scientifique globale.

9. http://www.dmgh.de/de/fs1/object/display.html?sortIndex=020:040, jusqu'en 829 (tomes 1 à $3)$; il faut, dans l'analyse, tenir compte du fait qu'il a bien davantage de conciles ( 2 tomes en 3 volumes) édités pour l'époque carolingienne que pour l'époque mérovingienne (1 tome).

10. MIGNE, PL, 104, col. 1068 (BM 632). Un autre diplôme est évoqué pour cette année-là par la chronique de Saint-Martin de Tours : les chanoines (canonici) auraient obtenu de l'empereur le 
droit d'élire librement leur abbé et le contrôle de l'élection abbatiale par les moines de leur dépendance de Cormery (et quod monachi Cormariacenses non possint eligere abbatem sine consensu canonicorum Sancti Martini Turonensis, inmo sint ei subiecti ; et quod canonici ex se ipsis eligant abbatem suum), cf. MGH, Scriptores, 26, éd. G. WAITZ, Hanovre 1882, p. 460.

11. MIGNE, PL, 104, col. 1214 (BM 909).

12. TESSIER, Recueil des actes..., op. cit., II, p. 224-226, n 328.

13. M. MOSTERT, «Les moines à Utrecht au temps de Willibrord", in Les moines dans la ville, éd. Histoire médiévale et archéologie, 7 (1996), p. 33-42 (p. 39-40).

14. MGH, Scriptores rerum merowingicarum, 2, éd. B. KRUSCH et W. LEVISON, Hanovre/Leipzig, 1937-1951, p. 489.

15. MGH, Scriptores rerum merowingicarum, 4, éd. B. KRUSCH, Hanovre/Leipzig, 1902, p. 202 et 205.

16. Lib. in Glor, $\S 47$, p. 70.

17. Liber Virt. sancti Iuliani, 17, p. 122.

18. La Règle de saint Benoît, 1, p. 440-441; la Règle du Maître, 1, 13, p. 332-333.

19. Vita Benedicti Anianensis (BHL 1096), AA SS, Feb. II, col. 612 et 614.

20. Vie des Pères du Jura, § 2, p. 238-239 (cellula peculiaris), § 128, p. 376-377 (cellula peculiaris) et $\S 173$, p. 424-425 (sens plus douteux : cellam, armarium, arcellam nullus illic omnino habuit nunquam).

21. Vie des Pères du Jura, § 37, p. 280-281 et $§ 39$, p. 282-283.

22. CASSIEN, Institutions, II, 2, p. 58-61.

23. La Règle du Maître, 1, 13, p. 332-334.

24. La Règle de saint Benoît, I, p. 438-441.

25. § 2, p. 238-239 et § 128, p 376-377 (claustro peculiaris cellae) ; § 1, p. 238-239 (claustra dans un sens métaphorique); § 141, p. 392-393 (claustra au sens de prison).

26. Vita Benedicti Anianensis (BHL 1096), AA SS, Feb. II, col. 614 : claustra nouo opere alia, cum columnis marmoreis quamplurimis, quce sitce sunt in porticibus.

27. $\$ 20$, p. 538-539 : in conventu tamen omnino breviatur oratio...

28. $\S 52$, p. 296-297 et $\S 129$, p. 378-379.

29. $\S 28$, p. 270-271 et $\S 170$, p. 422-423 (uno cunctos secum xenodochio quiescere fecit).

30. "Testament de Leodebaudus ", in M. PROU et A. VIDIER, Recueil des chartes de l'abbaye de SaintBenoit-sur-Loire, t. 1, Paris, 1907, p. 5.

31. Abbatiae officium (Lib. Vit. Patrum, 3, p. 285).

32. BHL 2825, MGH, Scriptores Rerum merowingicarum, 5, éd. B. KRUSCH, Hanovre/Leipzig, 1910, p. 203 et 206 .

33. Les autres occurrences viennent de mentions tardives de diplômes perdus et d'un diplôme faux attribué à Dagobert $\mathrm{I}^{\mathrm{er}}$.

34. Par exemple, dans un diplôme de Pépin le Bref de 752 : honus abbatiae (MGH, Diplomatum Karolinorum, I, éd. E. MÜHLBACHER, Hanovre, 1906, n² 2, p. 4-5).

35. Diplôme de Charlemagne, MGH, Diplomatum Karolinorum, I, éd. E. MÜHLBACHER, Hanovre, 1906, $n^{\circ}$ 91, p. 231-232.

36. MGH, Diplomatum Karolinorum, III, éd. T. SCHIEFFER, Berlin/Zürich, 1966, nº 8, p. 394-395.

37. MGH, Diplomatum Karolinorum, III, éd. T. SCHIEFFER, Berlin/Zürich, 1966, nº , 107, p. 253-256.

38. MGH, Concilia der Karolingischen Teilreiche 843-859, éd. W. HARTMANN, Hanovre, 1984, p. 433 ; notons, au passage, que cette abbatia n'est sans doute qu'une basilique desservie par des clercs et non un monastère.

39. Éd. GRAT et alii, p. 23.

40. Éd. GRAT et alii, p. 137.

41. Éd. GRAT et alii, p. 135. 


\section{RÉSUMÉS}

Cette enquête porte sur un certain nombre de mots (abbatia, casa, cella, cellula, claustrum, coenobium, congregatio, conventum, monasterium, xenodochium) qui sont susceptibles de désigner les communautés religieuses qu'elles soient monastiques ou canoniales. On a pu distinguer toute une série de termes dont les significations ne semblent pas changer $\mathrm{du} \mathrm{v}^{\mathrm{e}}$ au IX $\mathrm{IX}^{\mathrm{e}}$ siècle : des termes aux sens voisins monasterium et coenobium, des termes polysémiques, cella et cellula, servant à la fois à désigner le monastère et les pièces s'y trouvant, un terme employé rarement pour désigner un monastère, casa, avec une notion d'humilité. On a repéré également des termes désignant non les bâtiments mais les hommes (ou femmes) qui y vivent religieusement : congregatio avec un sens institutionnel fort et conventus, au sens institutionnel moins. Il existe également des termes pouvant être associés à un monastère mais ne permettant pas d'en affirmer l'existence, comme claustrum et xenodochium. Enfin une place particulière revient au mot abbatia, qui n'est jamais employé dans les règles étudiées mais est fréquent dans bien d'autres textes pour désigner la charge abbatiale; pour ce terme, on perçoit au IX siècle une évolution vers le sens actuel plus concret d'abbaye. Il convient donc d'examiner avec attention les contextes historiques et textuels de ces termes avant de les traduire ou de les utiliser pour caractériser une communauté religieuse ou le bâtiment qui l'abrite.

This study deals with a certain number of words (abbatia, casa, cella, cellula, claustrum, coenobium, congregatio, conventum, monasterium, xenodochium) that refer to religious communities regardless of the fact that they are monasteries or canonical houses. It is possible to distinguish a series of terms whose meanings obviously did not change from the fifth to the ninth century: the semantic field covered by the terms monasterium and coenobium; polysemic words like cella and cellula, designating not only the monastery, but also its rooms ; casa, a humble term rarely used to describe a monastery. We have also selected some terms which do not designate buildings, but men (or women) who led a religious life in those houses : congregatio with a strong institutional meaning and conventus, with a less institutional sense. There are also words like claustrum and xenodochium which can be associated with a monastery but which do not allow us to affirm the existence of a house. Finally, the term abbatia holds a special place : it is never used according to specific rules but in many texts it generally designates the office of an abbot; concerning this term, we have observed an evolution, since the ninth century, to the actual and more concrete meaning of abbey. For this reason, it is necessary to examine the historical and textual contexts of these terms carefully, before we translate or use them to characterize a religious community or its building.

\section{INDEX}

Mots-clés : monastère, abbaye, communauté religieuse

\section{AUTEUR \\ MICHÈLE GAILLARD}

Université de Lille, CNRS, UMR 8529-IRHiS 